\title{
T2* MRI Correlates with R2 Liver Iron Concentration in Transfusion Dependent Thalassaemia
}

\author{
Clarke $\mathrm{L}^{* 1}$, Kidson-Gerber $\mathrm{G}^{1}$, Moses $\mathrm{D}^{2}$, Wu GM-C ${ }^{1}$ and Lindeman $\mathrm{R}^{1}$ \\ ${ }^{1}$ Department of Haematology, Prince of Wales Hospital, Randwick, Sydney, NSW, Australia \\ ${ }^{2}$ Department of Radiology, Prince of Wales Hospital, Randwick, Sydney, NSW, Australia
}

${ }^{*}$ Corresponding author: Clarke L, Department of Haematology, Prince of Wales Hospital, Barker street Randwick, Sydney, NSW, Australia 2031, E-mail: lisaclarke84@hotmail.com

Citation: Clarke L, Kidson-Gerber G, Moses D, Wu GM-C, Lindeman R (2016) T2* MRI Correlates with R2 Liver Iron Concentration in Transfusion Dependent Thalassaemia. J Hematol Blood Disord 2(1): 102. doi: 10.15744/2455-7641.2.102

\section{Received Date: October 27, 2015 Accepted Date: January 14, 2016 Published Date: February 02, 2016}

\begin{abstract}
Background: Despite the introduction of chelation therapy, progressive iron overload continues to complicate management of transfusion dependent thalassaemia. Cardiac arrhythmias and cardiomyopathy are the predominant causes of death, while endocrinopathies and cirrhosis contribute to morbidity and mortality. Accurate assessment of iron is therefore critical in the long term management of this cohort.

Relaxometry based Magnetic Resonance Imaging (MRI) techniques have increasingly been adopted as a means of non invasive measurement of tissue iron concentration in patients with transfusion dependent thalassaemia. Currently, different MRI techniques are utilised to assess cardiac and hepatic iron concentrations, which correlate poorly. In an attempt to simplify the assessment of tissue iron concentration, we prospectively compared T2* MRI with R2 (FerriScan ${ }^{\oplus}$ ) derived liver iron concentration. A strong correlation exists between these two modalities, particularly at lower iron burdens. These findings would enable the concurrent assessment of cardiac and liver iron concentration thus streamlining total body iron assessment.

Method: 34 patients with $\beta$-thalassemia major underwent MRI assessment. R2 and T2* images were generated concurrently and R2 LIC calculated. Data was analysed with SAS/STAT 9.2 software.

Results: Statistical analysis demonstrated a positive linear relationship between T2* MRI and R2 LIC with a correlation coefficient (r) of $0.946, \mathrm{P}<0.001$. Based on this relationship a correlation equation of LIC $=36.745\left(\mathrm{~T} 2^{*}\right)^{-1.104}$ was generated and predicted T2* LIC values were calculated. Subsequent Bland-Altman analysis demonstrated a good agreement between the two methods with an estimated bias of -0.288 (95\%CI: -1.266, 0.690).

Conclusion: These results indicate there is a strong correlation between T2* MRI and R2 generated LIC. These findings have the potential to streamline iron assessment by facilitating the concurrent assessment of cardiac and liver iron concentration.

Keywords: Transfusion dependent thalassaemia; MRI T2*; MRI R2; Liver iron concentration; Transfusion related iron overload
\end{abstract}

\section{Introduction}

Regular transfusion in thalassaemia major results in progressive iron overload and its complications. Chelation therapy can prevent these complications and has been pivotal in the improved survival of these patients. With inadequate chelation, cardiac arrhythmias and cardiomyopathy are the predominant causes of death, while endocrinopathies and cirrhosis contribute to both morbidity and mortality [1-3]. Each chelating agent has potential risks and benefits. The accurate assessment of iron overload is therefore critical in guiding the appropriate choice of therapy and in managing adherence.

Serum ferritin is simple to perform and is useful in longitudinal monitoring but does not always correlate closely with iron overload. The liver iron concentration (LIC) has been validated as the best measure of total body iron stores but correlates poorly with cardiac iron burden, the strongest predictor of mortality. Magnetic Resonance Imaging (MRI) relaxometry can be used as a non invasive measurement of tissue iron and significant advances have been made with this technique over the past decade. Relaxation decreases in the presence of iron. Images are collected using magnetic gradients (T2*, R2*) or radio waves (T2, R2) and can be calculated as a function of time $(\mathrm{T})$ or expressed as a rate $(\mathrm{R})$, with the rate proportional to the inverse of time $\left(\mathrm{R} 2^{\star}=1000 / \mathrm{T} 2^{\star}\right)[3]$.

The R2 MRI method for determining LIC has been validated as a commercially available service (FerriScan ${ }^{\oplus}$ ) and has replaced liver biopsy for the evaluation of liver iron concentration and hence total body iron overload. Cardiac wall motion however renders R2 MRI unsuitable for the evaluation of cardiac iron loading. T2* MRI, with a shorter acquisition time capable of overcoming wall motion artifact, has been validated as a measure of iron loading in the intraventricular septum and as a predictor of cardiac complications of iron overload [4]. T2* MRI can also be used to measure hepatic iron loading. The major limitation to date is Liver T2* evaluation has not been validated as a measure of LIC to the same extent as the current "gold standard" R2 MRI, which has been calibrated and validated against liver biopsy, across a whole range of iron concentrations and in both paediatric and adult patients. 
In this study, we prospectively evaluated the accuracy and reproducibility of T2*MRI against R2 MRI generated LIC in patients with beta thalassemia major with a spectrum of transfusion related iron overload, as a surrogate validation of T2* LIC measurement.

\section{Methods}

34 patients (14 male, 20 female) with beta thalassemia major were included in the study (Mean age 31.9 \pm 6.88 years; 17-45years). All patients were transfusion dependent since childhood. Iron chelation regimens included deferiprone $(n=4)$, desferrioxamine $(n=6)$, deferasirox $(n=8)$ and combination therapy desferrioxamine and deferiprone $(n=16)$. Serum ferritin values were obtained monthly for the 6 months prior to the MRI scans with a wide range of levels obtained $(286-3295$ microg/L).

Ethics approval was obtained by the institution's ethics review committee and investigations were performed in accordance with the approved protocol. Formal written consent was obtained from all patients prior to enrolment.

\section{MRI method}

T2* and R2 images were simultaneously generated with a $1.5 \mathrm{~T}$ Siemen's Avanto MRI. T2* images were obtained via gradient echo sequence with $10 \mathrm{~mm}$ contiguous slices and echo times $1-20 \mathrm{~ms}, 12$ echos $(0.99 \mathrm{~ms}, 2.4 \mathrm{~ms}, 3.81 \mathrm{~ms}, 6.63 \mathrm{~ms}, 5.22 \mathrm{~ms}, 8.04 \mathrm{~ms}, 9.45 \mathrm{~ms}$ $10.86 \mathrm{~ms}, 12.27 \mathrm{~ms}, 15.09 \mathrm{~ms}, 13.68 \mathrm{~ms}, 16.5 \mathrm{~ms}$ ). CMRtools (Cardiovascular Imaging Solutions Ltd, London, UK) were used to generate $\mathrm{T}^{\star}$ values. The analysis was performed by a single radiologist and took approximately 45 minutes per patient.

R2 images were generated onsite (a validated radiology centre) and subsequently analysed by Resonance Health as per the FerriScan protocol $[5,6]$.

\section{Sampling method for $\mathrm{T} 2^{\star}$ images}

All nine segments of the liver were sampled and Regions of Interest (ROI) were established. The minimum diameter of the ROI was $20 \mathrm{~mm}$. Vessels, portal tract and areas of heterogeneity limited the maximal diameter. The ROI of each liver segment were divided into seven wedges. The $\mathrm{T} 2^{*}$ measurement for each wedge was calculated and averaged to obtain the $\mathrm{T} 2^{*}$ measurement of each ROI. The overall T2* was calculated by determining the mean of these values. Finally $\mathrm{T} 2^{\star}$ values were converted into $\mathrm{R} 2^{\star}$ values to facilitate comparison with R2 LIC.

\section{Statistical analysis}

Data was analysed using the SAS/STAT 9.2 software program and the relationship between R2* $\left(1 / \mathrm{T} 2{ }^{*}\right)$ and R2 LIC was modeled by ordinary least squares regression $(\mathrm{p}<0.05)$. Log transformation of R2* and R2 LIC was also applied and modeled in the same manner. Based on this relationship a correlation equation was generated and predicted LIC for corresponding T2* values were calculated. Finally a Bland- Altman plot was used to assess the agreement between R2 LIC and T2* predicted LIC.

\section{Results}

LIC values as measured by R2 ranged from 1.4 to $36.4 \mathrm{mg} / \mathrm{g}$ (mean 8.83 ), in a negatively skewed distribution. Mean liver T2* values ranged from 1.3 to $18.64 \mathrm{~ms}$ (mean 6.89) and Liver R2* values ranged from 53.65 to $769.23(1 / \mathrm{s})$ (mean 259.10) (Figure 1,2 and 3 ).

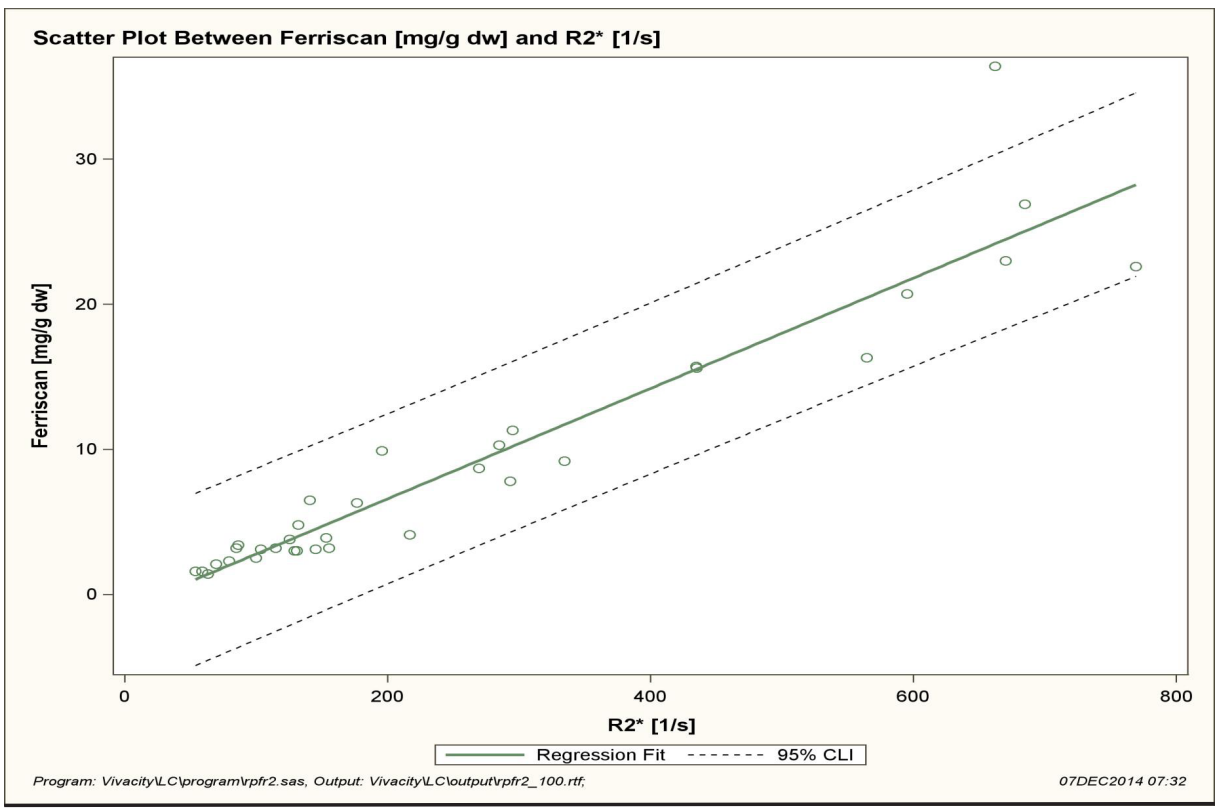

Figure 1: Shows the relationship between R2* and R2 LIC is close to linear with a correlation coefficient of 0.946 ( $p<0.001$ ). One data point is outside of the $95 \%$ CI of individual prediction values. Regression analysis: slope SE $=0.002,95 \%$ CI $0.033-0.043$, intercept SE $=0.772,95 \% \mathrm{CI}-2.586-0.557$ and $\mathrm{R}^{2}=0.894$ 


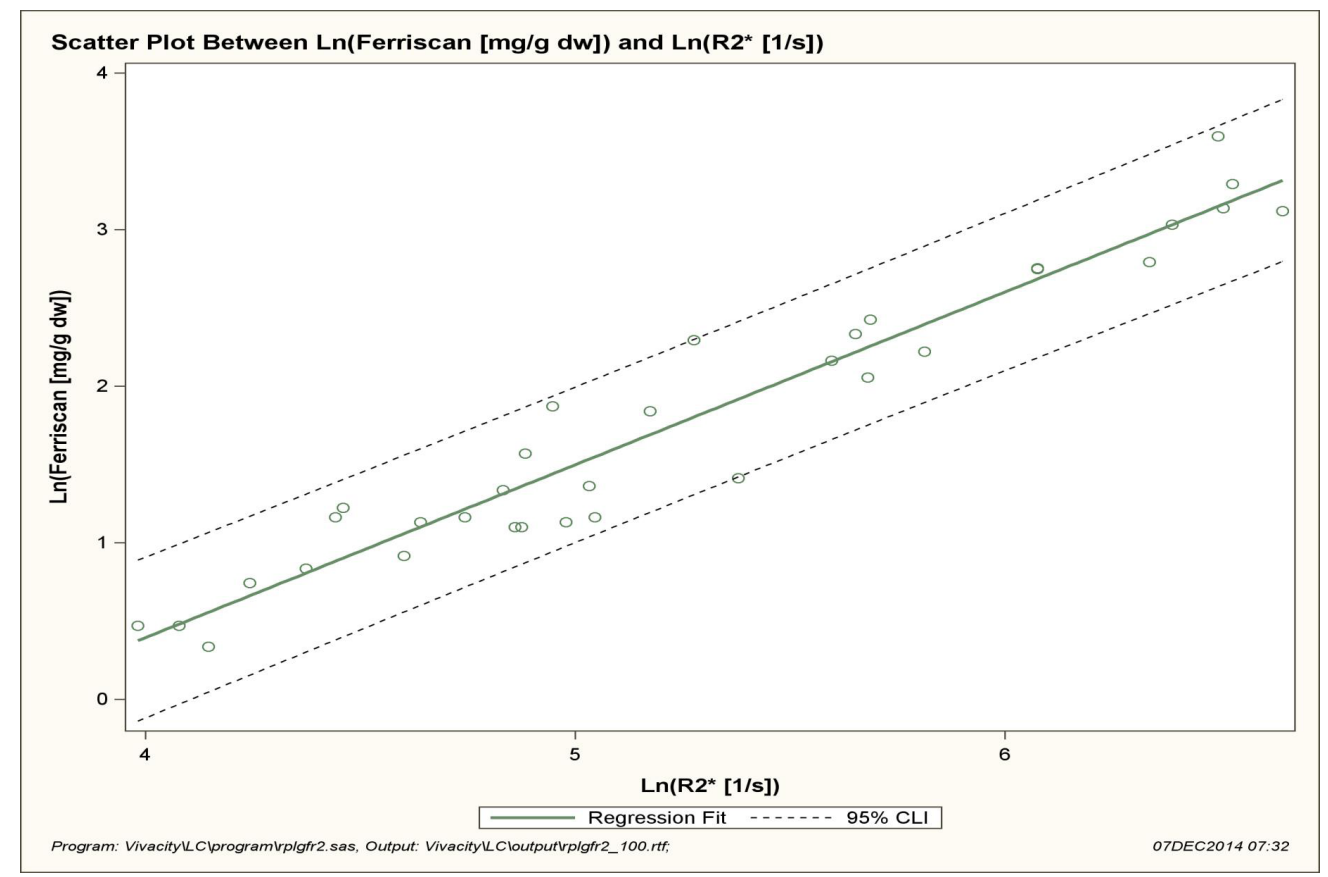

Figure 2: Shows the relationship between $\operatorname{Ln}\left(\mathrm{R} 2^{*}\right)$ and $\mathrm{Ln}(\mathrm{R} 2 \mathrm{LIC})$ is close to linear with a correlation coefficient of 0.965 ( $\left.\mathrm{p}<0.001\right)$ All data points are within the $95 \%$ CI of individual prediction values. Regression analysis: slope SE $=0.053,95 \%$ CI 0.997-1.212, intercept $\mathrm{SE}=0.280,95 \% \mathrm{CI}-4.593--3.454$ and $\mathrm{R}^{2}=0.9320$

These results demonstrate that a strong correlation exists between R2* MRI and R2 derived LIC. The outlying data point occurred in the severely iron overloaded population, a subset of patients where there is paucity of data in our study. The relationship appears more robust when log transformation of the data is performed. Based on this relationship a correlation equation of LIC=36.745 $\left(\mathrm{T} 2{ }^{\star}\right)^{-1.104}$ was generated.

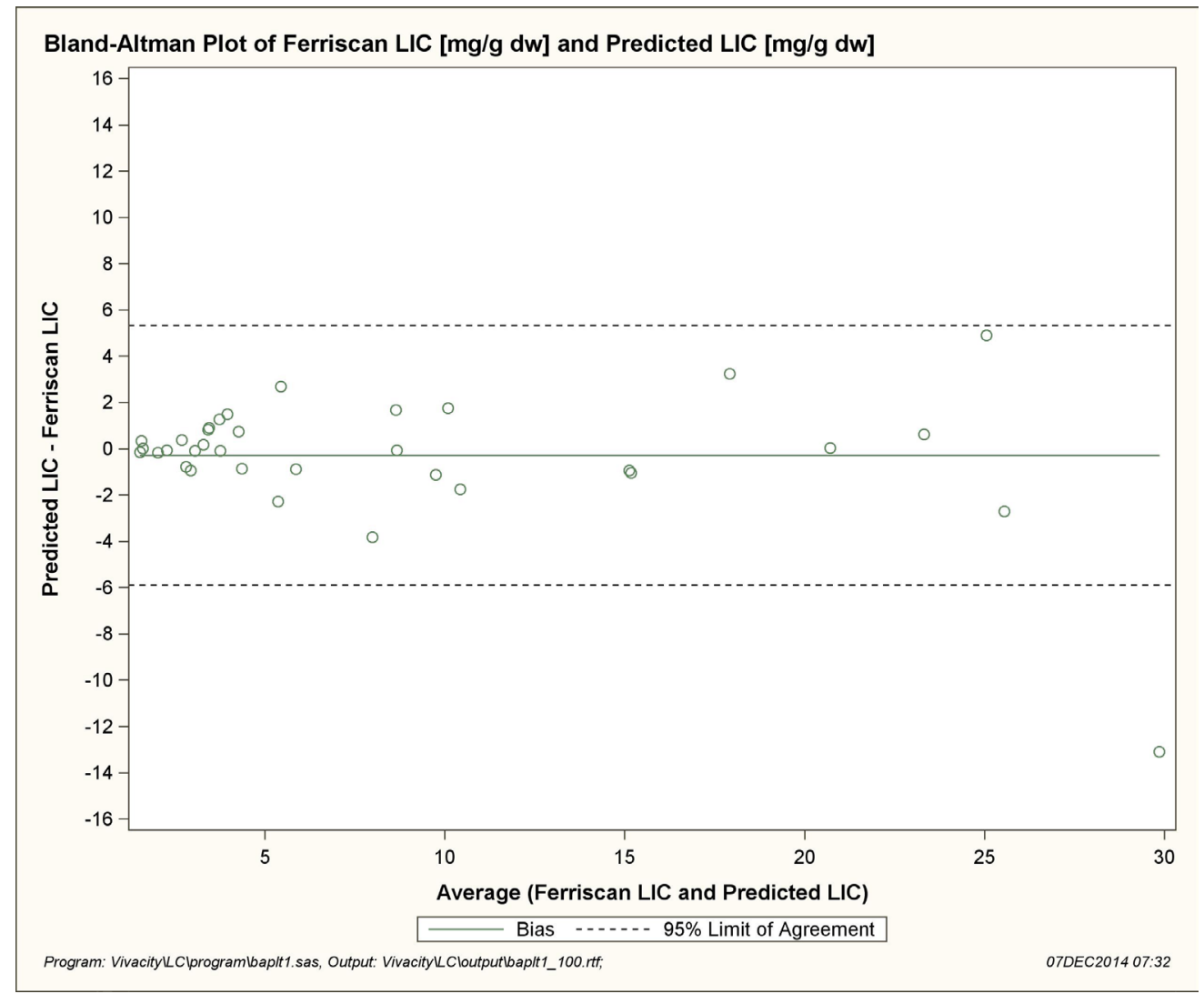

Figure 3: Shows the relationship between R2 generated LIC and T2* predicted LIC. Only one data point is outside of the $95 \%$ limit of agreement $(-5.90,5.32)$. The agreement between the two measures is relatively good with an estimated bias of $-0.288(95 \%$ CI: -1.266 , $0.690)$ 


\section{Discussion}

The major benefits of utilising T2* MRI for the assessment of LIC include shorter acquisition times and concurrent assessment of liver and cardiac iron burden, features that are particularly beneficial in the paediatric population. Current limitations of this methodology include lack of standardisation, interference due to external magnetic properties and an inability to quantify iron burden in heavily iron overloaded patients (dry weight $>25 \mathrm{mg} / \mathrm{g}$ ) [7].

Validation of Liver T2* measurements against biopsy determined LIC has been documented by several groups [7-11] however there appears to be limited concordance between the studies. It has been hypothesised that inherent limitations of earlier MRI acquisition techniques, lack of methodology standardisation or sampling bias may have contributed to these inconsistencies. More recently several groups have demonstrated a correlation between R2 and R2* derived LIC. Garbowski et al directly compared T2* MRI with biopsy and indirectly against R2 generated LIC [11]. Chan et al has performed a MRI retrospective analysis [12] and Wood et al prospectively compared R2 and R2* derived LIC in both Thalassaemia major and Sickle cell disease patients [10]. These studies observed a close relationship between R2* and R2 LIC however the relationships were not as robust as demonstrated in our study.

The determination of LIC via T2* MRI traditionally involves acquisition of data from a single to several transverse slices through the liver histological studies reporting intra organ variability in liver iron concentration measurements performed on biopsies from despite livers [13]. Meloni et al demonstrated that liver T2* variations were limited and when present they were secondary to artifact or measurement variability [14]. In an attempt to negate the presence of any sampling bias, we calculated average T2* values obtained from nine ROI, divided into seven wedges. Whilst this method appears to examine greater ROI compared to other studies, regional analysis failed to reveal significant differences in T2* values. Whilst this finding supports Meloni's results suggesting that only a single ROI need to be interrogated it must be noted that there was no evidence of cirrhosis in our population, which may have contributed to the concordant results.

This prospective study analysed a homogenous group of patients, all with transfusion thalassaemia major, and evaluation avoided variations in liver biopsy LIC by comparing MRI attained results only. These factors all differ from previous studies and may explain the greater degree of correlation achieved in this study.

One data point fell outside the 95\% CI of individual prediction values in the direct comparison of R2 derived LIC and T2* values. This occurred in the severely iron overloaded population, consisting of only a limited subset of patients. As such it is difficult to draw any conclusions as to possible contributing factors. Interestingly the corresponding patient's ferritin, at the time of MRI assessment, was only $1314 \mathrm{microg} / \mathrm{L}$ (range 286-3295microg/L) further highlighting the limitations of using ferritin to monitor body iron stores.

The major limitation of this study is the paucity of data for LIC $>10 \mathrm{mg} / \mathrm{g}$ dry weight, particularly LIC $>25 \mathrm{mg} / \mathrm{g}$. This population appears to be prone to the greatest degree of discrepancy in all validation studies. Assessment of patients falling into the category of severe iron overload is important because whilst cardiac and liver iron concentrations are often discordant, LIC $>15 \mathrm{mg} / \mathrm{g}$ have been found to correlate with increased risk of cardiac disease and premature death [15]. However, whilst it is crucial to identify those patients with high LIC in order to implement aggressive chelation therapy, accurate assessment of LIC is more relevant in patients with lower LIC to facilitate chelation dose modifications and thus avoid excessive exposure and unnecessary toxicity. Further limitations of this study include a small sample size in a single site with only a single radiologist involved in the analysis of the $\mathrm{T}^{*}$ images.

\section{Conclusion}

This study demonstrates that there is a good correlation between T2* MRI and R2 generated LIC especially in patients with LIC $<10 \mathrm{mg} / \mathrm{g}$ dry weight. As the study population is reflective of the wider transfusion dependent thalassaemia population, these findings have the ability to directly influence current clinical practice by facilitating the use of a single MRI for the concurrent assessment of cardiac and liver iron burden thus simplifying the assessment body iron stores. Whilst this relationship has been documented previously, the methodology adopted in this study demonstrates the most robust relationship. At present due to the paucity of data in heavy iron overloaded patients, the simplification of tissue iron assessment with a single T2* MRI should be limited to patients with LIC $<10 \mathrm{mg} / \mathrm{g}$ dry weight. Future studies incorporating larger numbers, across multiple centers, with different analysts would be beneficial, particularly in an attempt to address the paucity of data in the more heavily iron loaded population.

\section{Acknowledgments}

RL designed this study. DM performed data analysis. GMC abstracted the data. LC led writing of the manuscript. GKG critically appraised the manuscript. No funding was received to perform this study and the authors deny any conflict of interest.

\section{References}

1. Ho PJ, Tay L, Lindeman R, Catley L, Bowden DK (2011) Australian guidelines for the assessment of iron overload and iron chelation in transfusion-dependent thalassaemia major, sickle cell disease and other congenital anaemias. Intern Med J 41: 516-24. 
2. Echeverria JMA, Castiella A, Emparanza JI (2012) Quantification of iron concentration in the liver by MRI. Insights Imaging 3: $173-80$.

3. Wood, JC (2011) Impact of iron assessment by MRI. Hematology Am Soc Hematol Educ Program 2011: 443-50.

4. Anderson LJ, Holden S, Davis B, Prescott E, Charrier CC, et al. (2001) Cardiovascular T2-star (T2*) magnetic resonance for the early diagnosis of myocardial iron overload. Eur Heart J 22: 2171-9.

5. St Pierre TG, Clark PR, Chua-Anusorn W (2004) Single spin-echo proton transverse relaxometry of iron-loaded liver. NMR Biomed 17: 446-58.

6. St Pierre TG, Clark PR, Chua-anusorn W, Fleming AJ, Jeffrey GP, et al. (2005) Noninvasive measurement and imaging of liver iron concentrations using proton magnetic resonance. Blood 105: 855-61.

7. Christoforidis A, Perifanis V, Spanos G, Vlachaki E, Economou M, et al. (2009) MRI assessment of liver iron content in thalassamic patients with three different protocols: comparisons and correlations. Eur J Haematol 82: 388-92.

8. Vasanawala SS, Yu H, Shimakawa A, Jeng M, Brittain JH, et al. (2012) Estimation of liver $\mathrm{T}_{2}$ in transfusion-related iron overload in patients with weighted least squares $\mathrm{T}_{2}$ IDEAL. Magn Reson Med 67: 183-90.

9. Hankins JS, McCarville MB, Loeffler RB, Smeltzer MP, Onciu M, et al. (2009) R2* magnetic resonance imaging of the liver in patients with iron overload. Blood 113: 4853-5.

10. Wood JC, Enriquez C, Ghugre N, Tyzka JM, Carson S, et al. (2005) MRI R2 and R2* mapping accurately estimates hepatic iron concentration in transfusiondependent thalassemia and sickle cell disease patients. Blood 106: 1460-5.

11. Garbowski MW, Carpenter JP, Smith G, Roughton M, Alam MH, et al. (2014) Biopsy-based calibration of T2* magnetic resonance for estimation of liver iron concentration and comparison with R2 Ferriscan. J Cardiovasc Magn Reson 16: 40.

12. Chan WC, Tejani Z, Budhani F, Massey C, Haider MA (2014) R2* as a surrogate measure of ferriscan iron quantification in thalassemia. J Magn Reson Imaging 39: $1007-11$.

13. Emond MJ, Bronner MP, Carlson TH, Lin M, Labbe RF, et al. (1999) Quantitative study of the variability of hepatic iron concentrations. Clin Chem 45: 340-6.

14. Meloni A, Luciani A, Positano V, De Marchi D, Valeri G, et al. (2011) Single region of interest versus multislice T2* MRI approach for the quantification of hepatic iron overload. J Magn Reson Imaging 33: 348-55.

15. Wood JC (2014) Guidelines for quantifying iron overload. Hematology Am Soc Hematol Educ Program 2014: 210-5.

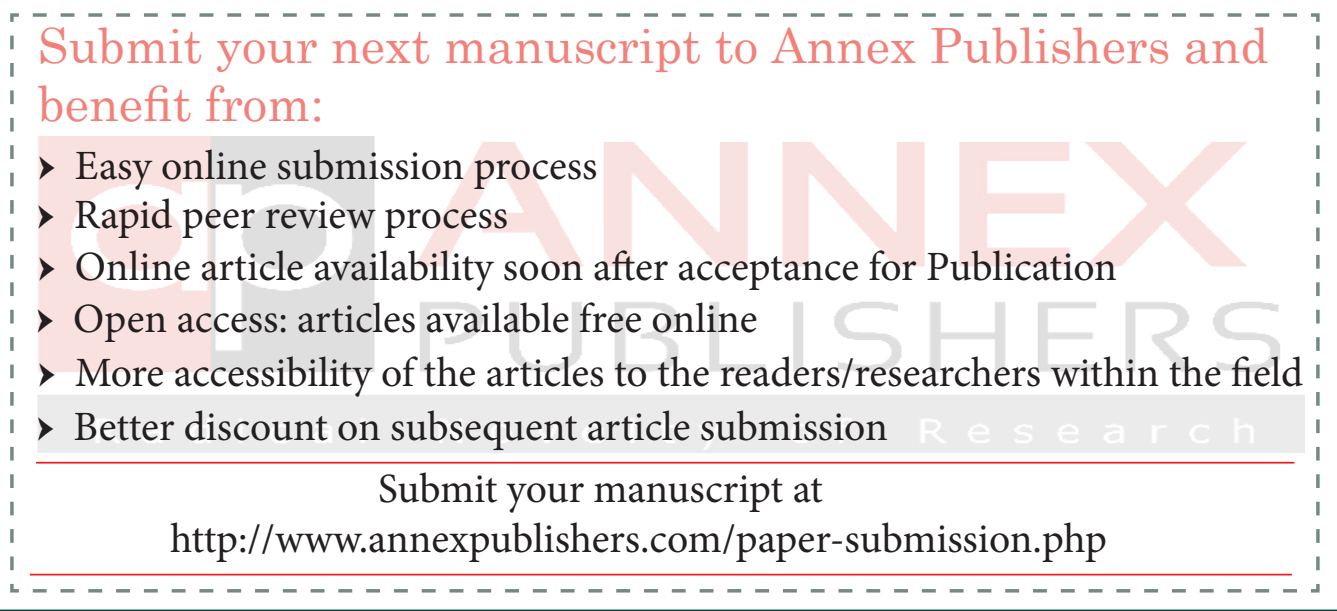

